\title{
Topic Detection and Evolution Analysis on Microblog*
}

\author{
Guoyong Cai, Libin Peng, and Yong Wang \\ Guilin University of Electronic Technology, Guangxi Key Lab of Trusted Software, \\ 541004 China \\ ccgycai@guet.edu.cn, ccsupeng@163.com, hellowy@126.com
}

\begin{abstract}
The study on topic evolution can help people to understand the ins and outs of topics. Traditional study on topic evolution is based on LDA model, but for microblog data, the effect of this model is not significant. An MLDA model is proposed in this paper, which takes microblog document relation, topic tag and authors relations into consideration. Then, the topic evolution in content and intensity is analyzed. The experiments on microblog data have shown the effectiveness and efficiency of the proposed approach to topic detection and evolution analysis on Microblog.
\end{abstract}

Keywords: Microblog, LDA model, Topic Evolution, Topic Detection.

\section{Introduction}

Microblog is the shortened form of micro blog (MicroBlog). It is a type of platform which allows users to disseminate/obtain/share information based on relationship among users. Users can set up personal community through WEB, WAP and other client terminals, update the information with no more than 140 words of text, and achieve instantly information sharing. However, the content of microblog is diverse and changing rapidly. It is a challenging problem of how to discover automatically an effective topic and to analyze the evolution of the discovered topic.

Topic is defined as a number of related events caused by a set of seed in Topic Detection and Tracking[1]. The topic model represented by LDA (Latent Dirichlet Allocation) [2] is an important technology in the field of text mining in recent years. LDA model has a good ability of dimension reduction and scalability. It has achieved great success in mining traditional network news topic. Topic evolution is referred to the migration of topic content and intensity over times[3]. Researches on topic evolution in general are based on LDA model to extract topics, and then the topic evolution of content and intensity are analyzed. However, compared with the traditional network text, Microblog has distinct characteristics, such as a short text (usually less than 140 characters), sparse data, noise data, mixed and disorder content, etc. Besides, there also exist social relations, structural social network information. Therefore, it is not effective to use LDA model for microblog text. An MLDA model is proposed in this paper, which takes microblog document relation, topic tag and authors' relationships into consideration. The topic evolution in content and intensity are analyzed based on MLDA model.

\footnotetext{
* This work is supported by the NSFC(\#61063039), Guangxi Key Lab of Trusted Software (\#kx201202).
} 


\section{Related Works}

Due to its good scalability, LDA model, which proposed by Blei in 2003[2], is extended by many scholars. Researches on topic evolution based on the scalable LDA model are divided into the following three categories:

(1) Continuous time models. The information of time is taken into topic model as a variable to study the topic evolution. For example, a TOT (Topic Over Time) model is proposed by Wang [4]. In TOT model, each document is a mixture of topics that is influenced jointly by its time stamp. The main disadvantage of TOT is that it uses the beta distribution to model the topic development trends. Therefore, for documents that don't release time, it will predict the release date of the document. For documents with given released date, it can predict the document distribution.

(2) Pre-discretization methods. In this type of method, documents are divided into some parts according to the time windows before modeling topics, and then documents are processed and the topic distribution is generated in each time window. Song and etc.[5] proposed a ILDA model (Incremental Latent Dirichlet Allocation) to study the content evolution of a topic and solve ILDA model with Gibbs sampling approach[6]. Online LDA model is proposed by AlSumait[7], history data are used as the prior distribution of the proposed model and LDA model are used to study topic evolution for the arrived data in each time interval. Hu Yanli and etc. [9] have also implemented the online topic evolution analysis based on online LDA model, but they consider the inter-connection of topic distribution in each time slice.

(3) Post-discretization methods. In this type of method, the affect of time is not considered first. After topics are extracted based on LDA in the document set, the topics are divided into time slice according to its time stamp. Grillffiths and Steyvers[8] have proposed a post-discretization method based on LDA model, they use the intensity of topics in each time slice to indicate the topic trends.

\section{Topic Evolution Analysis on Microblog}

\subsection{LDA Model}

LDA model is a three-layer probability model. It consists of word, topic and document. The key idea behind the LDA model is to assume that the words in each document were generated by a mixture of topics, where a topic is represented as a multinomial probability distribution over words[9]. Each document has a specific mixture of topics, which generated from the Dirichlet distribution. The specific idea behind LDA is to assume that each document correspond to the multinomial distribution $\theta$ of $T$ topics, each topic correspond with the multinomial distribution $\varphi$ of $N_{d}$ words, $\theta$ is the prior of Dirichlet distribution with parameters $\alpha, \varphi$ is the prior of Dirichlet distribution with parameters $\beta$. For each word in a document $d$, topic $z$ is extracted from the distribution $\theta$ of the document, and the word $w$ is extracted from the distribution $\varphi$ of topic $z$. This process will be repeated $N_{d}$ times, then the document $d$ is generated. The generative process of LDA is shown in figure1. 


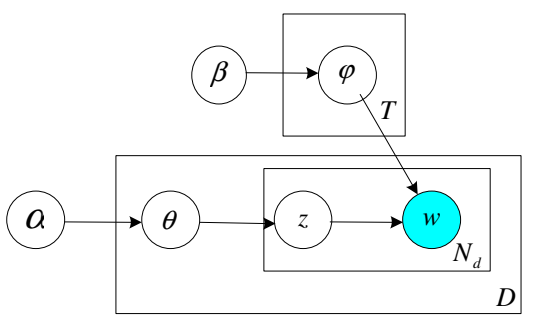

Fig. 1. LDA model

The shaded circles in Figure 1 represent the observed variable and the unshaded circles represent the latent variables. The arrows between two variables represent the conditional-dependence and boxes represent repeated sampling, the number of repetitions is located at the lower right corner of the box. There are two parameters need to be inferred in this model, one is the distribution $\theta$ of document-topic and the other is the distribution $\varphi$ of topic-word. Since it is difficult to obtain precise parameters, VEM[2], Gibbs Sampling[6] and Expectation propagation[10] are often applied to estimate the parameters. Gibbs Sampling has been widely used for its simple implementation.

\subsection{Topic Discovery Model ----MLDA Model}

Compared with other texts, microblog texts have special symbols, such as "@"、"\# " and "retweet". @ indicates the author's relationship of a microblog post. For example, a message "@Sandy Congratulations, you get a good job." and another message "@Sandy Can you teach me some IT knowledge used in a work". When considering the author's relationship, we can set a connection between these two seemingly unrelated microblogs and consider that "job" in the first message is related to "IT" in the second message. \# indicates topic tag in a microblog. For example a message "Sunyang, come on! \#Olympic Games\#". If considering the topic tag, "Sunyang" and "Olympic Games" are related. "retweet" indicates microblog documents' relation. For example, a message "Chinese Dream, retweet @ Sandy the best popular new vocabulary ...". It is difficult to analyze the specific meaning for "Chinese Dream", but compared with the original microblog, we know that "Chinese Dream" is a kind of "new vocabulary".

An MLDA model, extended from LDA, is proposed in this paper, which takes microblog document relation, topic tag and authors' relationships into consideration. The parameters of MLDA model is shown in table 1. The Bayesian network of MLDA is showed in figure 2. $c$ indicates the author's relationship, $\alpha_{r}$ indicates microblog document relation. $t$ indicates topic tag in mircoblog. $\alpha_{c}$ is the parameter of distribution, $\theta_{c}$ associate with authors' relation. $\theta_{c}$ is computed by Dirichlet distribution with parameter $\alpha_{c} . \alpha$ is the parameter of distribution $\theta_{d}$ of document-topic. $\alpha_{t}$ is the parameter of distribution $\theta_{d}$ associate with special topic. 


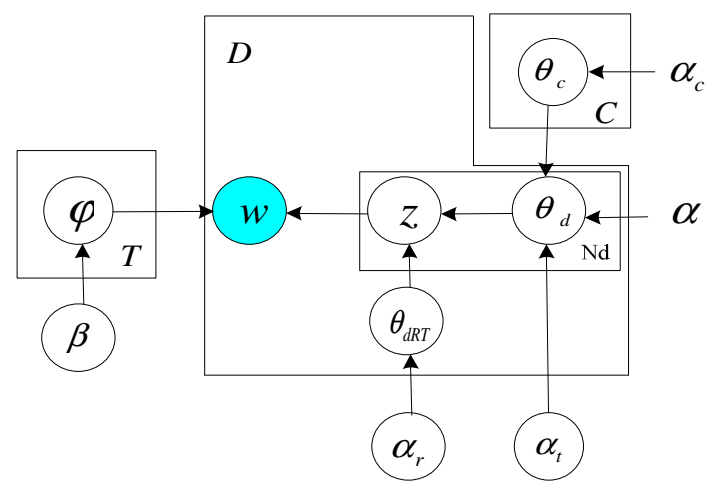

Fig. 2 Bayesian network of MLDA

$\alpha_{r}$ is the parameter that decide whether it is a retweet message. The distribution $\theta$ of topics in microblog is shown as follow.

$$
P\left(\theta \mid \alpha, \alpha_{c}, \alpha_{t}, \pi_{c}, t\right)=\left[P\left(\theta_{c} \mid \alpha_{c}\right)^{\pi_{c}} P\left(\theta_{d} \mid \alpha\right)^{1-\pi_{c}}\right]^{1-t} P\left(\theta_{d} \mid \alpha_{t}\right)^{t}
$$

If there exists a symbol "@" in microblog, the value of $\pi_{c}$ is 1 , otherwise $\pi_{c}$ is 0 . If there exists a symbol "\#" in microblog, boolean variable $t$ is 1 , otherwise $t$ is 0 . If there exists "retweet", the distribution of $z_{d, i}$ over topics depend on $\theta_{d R T}$ and $\theta_{d}$, sampling from a Bernoulli distribution with parameter $a_{r}$ to decide $\theta_{d R T}$ and $\theta_{d}$, and based on them we extract the current topic $z_{d, i}$. Otherwise, we extract topics $z_{d, i}$ directly from Multinomial distribution with parameter $\theta_{d}$.

For a microblog, the joint probability distribution over all words and their topics is computed as following equation (2).

$$
\begin{aligned}
& P\left(w, z \mid \alpha_{r}, \theta, \beta\right)=P\left(\theta_{d R T} \mid \alpha_{r}\right) P(z \mid \theta) P(w \mid z, \beta) \\
& =P\left(\theta_{d R T} \mid \alpha_{r}\right) P\left(z \mid \theta_{d}\right)^{1-\alpha_{r}} P\left(z \mid \theta_{d R T}\right)^{\alpha_{r}} P(w \mid z, \beta)
\end{aligned}
$$

The description of generative process in MLDA is as follows:

(1) For each document $d \in D$, if there exists "\#topic\#", we compute $\theta_{d}=\theta_{d} \sim \operatorname{Dri}\left(a_{t}\right)$. If there exists a symbol "@", we compute $\theta_{d}=\theta_{c} \sim \operatorname{Dir}\left(\alpha_{c}\right)$. In other cases, we compute $\theta_{d}=\theta_{d} \sim \operatorname{Dir}(\alpha)$.

(2) For each topic $z_{d, i}$, we judge document relation with "retweet". If there exists "retweet", then $z_{d, j} \sim \operatorname{Multiomial}\left(\theta_{d R T}\right)$. Based on $z_{d, i}$, we generate $\varphi \sim \operatorname{Dir}(\beta)$.

(3) For each word $w_{d, i}$ in a document, firstly, we choose a topic $z_{d, j} \sim \operatorname{Multiomial}\left(\theta_{d}\right)$; and then we choose a word with $w_{d, j} \sim \operatorname{Multiomial}(\varphi)$. 
Table 1. Parameters in MLDA

\begin{tabular}{|c|l|}
\hline Parameter & \multicolumn{1}{c|}{ Definition } \\
\hline $\begin{array}{c}\alpha, \alpha_{t} \\
\alpha_{c}, \beta^{t}\end{array}$ & The prior parameters of $\theta_{c}, \theta_{d}, \varphi$ \\
\hline$\theta_{c}$ & Topic distribution associate with authors' relation \\
\hline$\theta_{d}$ & Topic distribution over microblog $d$ \\
\hline$\varphi$ & The distribution of topic-word \\
\hline$z_{d, i}$ & The $i$ th topic in document $d$ \\
\hline$w_{d, i}$ & The $i$ th word in document $d$ \\
\hline$D$ & The number of documents \\
\hline$T$ & The number of topics \\
\hline$N_{d}$ & The number of words \\
\hline$\pi_{c}$ & $\begin{array}{l}\text { Bool parameter, decide whether there exists a @ message. If } \\
\text { so, } \pi_{c} \text { is 1, else } \pi_{c} \text { is } 0 .\end{array}$ \\
\hline$\alpha_{r}$ & Decide whether it is a retweet message \\
\hline
\end{tabular}

\subsection{Algorithm Implementation}

We apply Gibbs sampling to estimate the parameters $\theta, \varphi$. Gibbs sampling is one simple realization method of MCMC (Markov chain Monte Carlo), which is a fast and effective algorithm for estimating the parameters[11]. Gibbs sampling is based on posterior distribution of words given a topic $p(z \mid w)$. Repeated iterations on the probability distribution, the parameters are derived. The process is computed with equation (3).

$$
P\left(z_{i}=j \mid z_{-i}, w_{d, i}, \alpha, \beta\right) \propto \frac{n_{-i, j}^{(w)}+\beta}{n_{-i, j}^{(.)}+N_{d} \beta} * \frac{n_{-i, j}^{(d)}+\alpha}{n_{-i, .}^{(d)}+N_{d} \alpha}
$$

Where, $n_{-i, j}^{(w)}$ is the word counts assigned to topic $j$ and $w . n_{(-i, j)}^{(.)}$is the counts of words assigned to topic $j . n_{-i, j}^{(d)}$ is the counts of words assigned to topic $j$ in document $d . n_{-i, \text {, }}^{(d)}$ is the counts of words assigned to topic $j$. All counts do not include the current iteration. After iteration finished, we can estimate $\theta$ and $\varphi$ from the value $z$ using equation (4).

$$
\theta=\frac{n_{j}^{(d)}+\alpha}{n_{.}^{(d)}+T \alpha} \quad \varphi=\frac{n_{j}^{(w)}+\beta}{n_{j}^{(.)}+N_{d} \beta}
$$


Where, $n_{j}^{(d)}$ denotes the number of words that assigned to topic $j$ in document $d . n^{(d)}$ denotes the number of words appeared in document $d . n_{j}^{(w)}$ denotes the number that word $w$ is assigned to topic $j . n_{j}^{(.)}$is the number of words that assigned to topic $j$.

The Gibbs sampling procedure of MLDA is implemented and shown in Fig.3. The Gibbs sampling algorithm consists of three parts: initialization, burn-in and sampling.

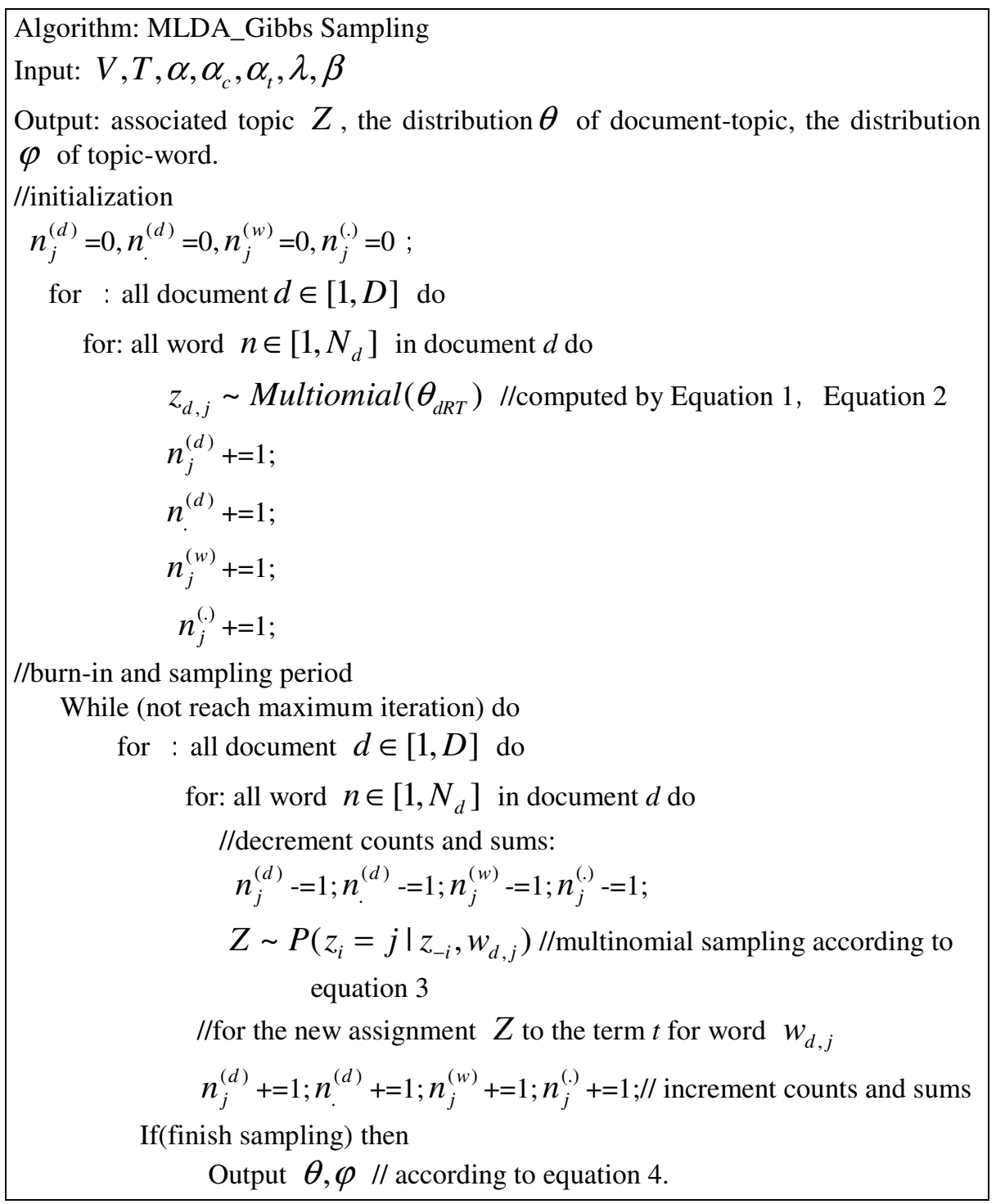

Fig. 3. Gibbs sampling algorithm 


\subsection{Topic Evolution}

Topic evolution indicates that the same topic shows dynamism and difference with time going. The evolution of topic reflects in two aspects. First, the topic intensity changes over time. For example, the topic is the Olympic Games which take place every four years, which are active for Olympic years, other years is low. On the other hand, the topic content changes with the passage of time. For example, on the eve of the Olympic Games, when we pay more attention to the Olympic preparations, on the middle of Olympic, gold medal topics will be focused. In the end of Olympic Games, more attention should be paid to the summary and inventory of the Olympic Games. We use the distribution of topic $j$ with time $t$ to define the intensity of topic.

$$
\delta_{j}^{t}=\frac{1}{D^{t}} \sum_{d \in D^{t}} \theta_{d, j}
$$

Where $\delta_{j}^{t}$ is the intensity of a topic, $D^{t}$ is the number of document, $\theta_{d, j}$ is the distribution of topic $j$.

The content of topic evolution is characterized as the degree associated with topics. The differences of the distribution of two topics is described with $\mathrm{KL}$ (Kullback-Leibler) distance[12]. The smaller the difference is, the higher degree of the two topics will be associated. Assuming that the probability distribution of topic $\mathrm{A}$ is $A=\left(A_{1}, A_{2} \ldots, A_{n}\right)$, and $\mathrm{B}$ is $B=\left(B_{1}, B_{2} \ldots, B_{n}\right)$, the KL distance between topic $\mathrm{A}$ and $\mathrm{B}$ is computed as follow.

$$
K L(A, B)=D(A \| B)=\sum_{i}^{N} A_{i} \log \frac{A_{i}}{B_{i}}
$$

\section{$4 \quad$ Experiments}

The experimental data are captured from a large microblog site named Sina microblog. Through API provided by Sina, about 8 million original microblog data from July $1^{\text {th }}$ 2012 to August $29^{\text {th }} 2012$ are captured. We mark five topics, i.e., "London Olympics", "heavy rains in Beijing", "Price War", "Diaoyu Island Event", "The Voice of China". In order to save space or to speed up sampling, the punctuation and stop words in the original microblog dataset must be removed before experiments. We complete this preprocessing work by using a punctuation list and a stop words dictionary.

\subsection{Topic Discovery}

Effectiveness experiments are conducted on dataset mainly to examine the performance of MLDA. The parameters are set up with $\alpha=0.2, \alpha_{c}=\alpha_{t}=\lambda=1$, $\beta=0.1, T=5, N_{d}=716$. The key words of each topic are extracted by MLDA models. The results are shown in table 2 . 
Table 2. The list of representative words for each topic

\begin{tabular}{|c|c|}
\hline topics & Most representative words \\
\hline Topic1: London Olympic & $\begin{array}{c}\text { Olympic Games, participation, London, } \\
\text { champion, gold metal }\end{array}$ \\
\hline Topic2: Heavy rains in Beijing & $\begin{array}{c}\text { heavy rains, disaster, rescue, flood protection, } \\
\text { emergency }\end{array}$ \\
\hline Topic3: Price War & $\begin{array}{c}\text { Sunning, jingdong, price war, } \\
\text { E-commerce, fund }\end{array}$ \\
\hline Topic4: Diaoyu Island Event & $\begin{array}{c}\text { Diaoyu Island, Japan, China, Sovereignty, } \\
\text { protection }\end{array}$ \\
\hline Topic5: The Voice of China & Voice, China, music, pleasant music, jury \\
\hline
\end{tabular}

\subsection{Intensity Evolution of Topics}

We analyse the topics from July to August in 2012. The different change of the intensity of topics will be calculated in different times, which is divided into eight time slice by each a week. The intensity of each topic is shown in figure 3.The ordinate represents the intensity of the topic at each time slice, which is calculated with equation 5.

Figure 3 shows us that the intensity of topic 1 is higher than other topics during each time slice. This demonstrates that the influence of topic 1 is higher and is a hot topic during the two months. Besides, for each topic, the intensity of topics will change with time. This shows that each topic will go through a evolutional process that is consistency with reality.

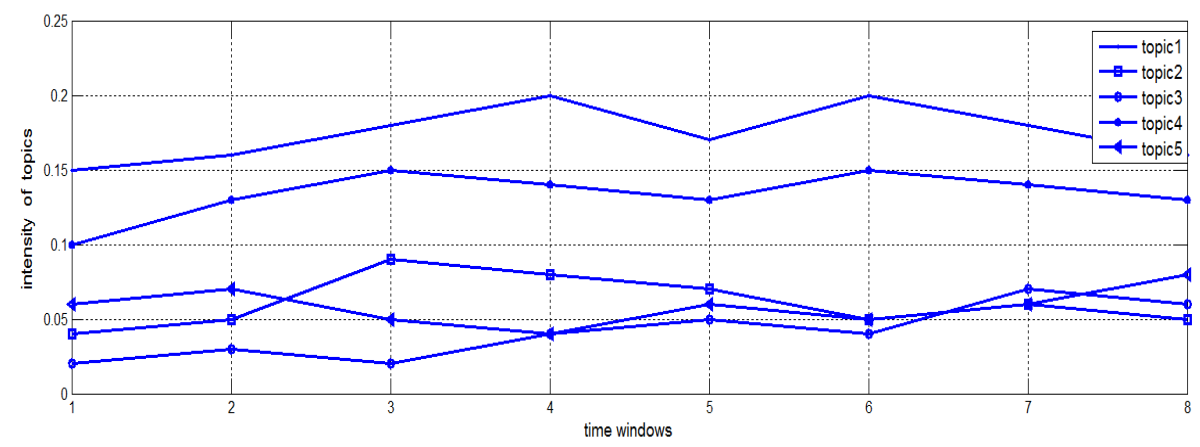

Fig. 4. The intensity evolution of topics

\subsection{Content Evolution of Topics}

For topic 1, the intensity is higher over time. The similarity distance of a topic during the adjacent time slice indicates the content evolution of the topic. KL distance is calculated according to the probability distribution of words in eight time intervals. Figure 4 presents the KL distance of topic 1 changes over the intervals, which indicates 
that the content of the topic changes over intervals. As can be seen from the figure 4 that the KL distance of topic 1 enlarge abruptly during the time slice 4 and 5, which indicates that the content of topic 1 changes quite a few. Figure 5 shows that the content of topic 1 have changed from "gold metal" into "open ceremory". During time slice 6 and7, the KL distance enlarge abruptly, which indicated that the content of topic1 have changed into "closing ceremony". The topic changing process is shown in figure 5.

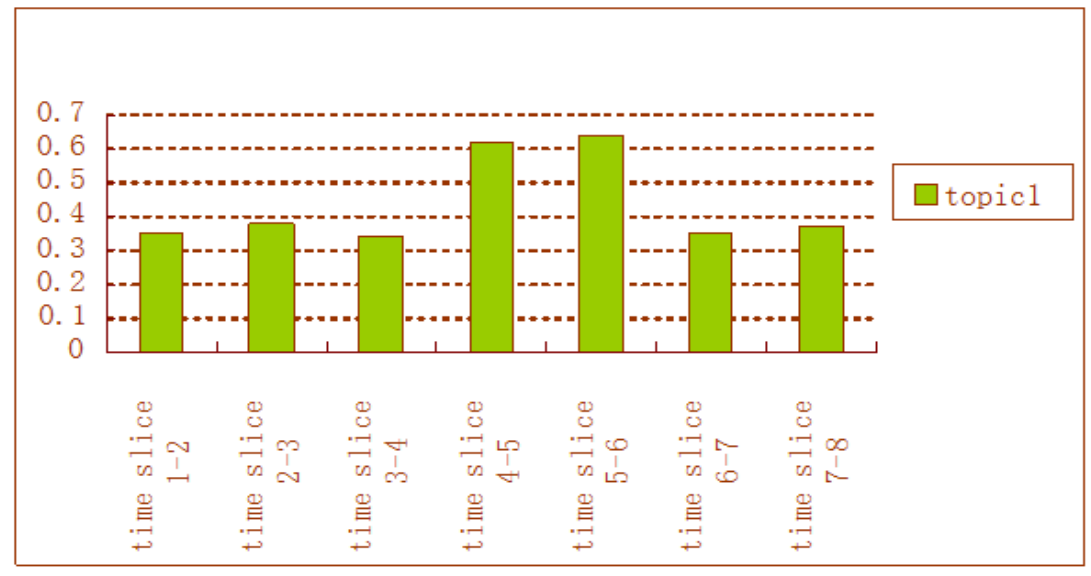

Fig. 5. The KL distance of topic 1

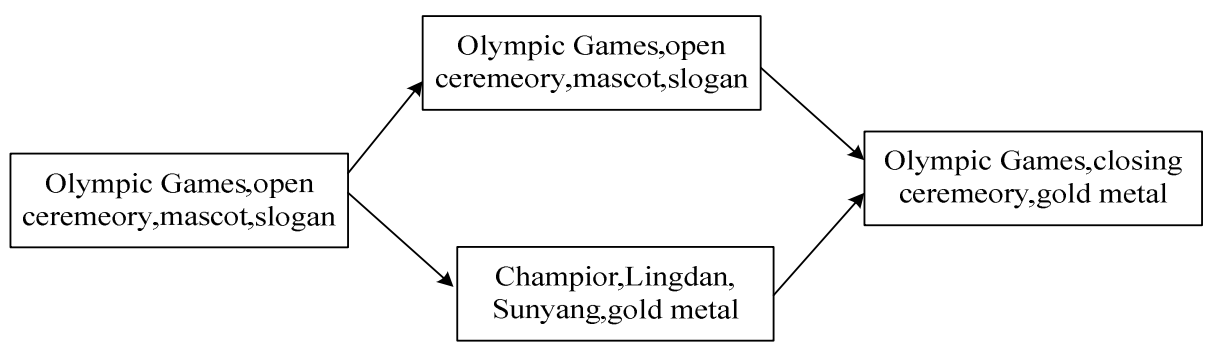

Fig. 6. The chart of topic 1 evolution

In summary, the trend of topic evolution can be captured quite accurately with MLDA model. It indicates not only the intensity change of the topic over time, but also the content change of the topic over time.

\subsection{Comparisons of Algorithm Performance}

We conduct a comparative experiment between MLDA and LDA, which is a baseline model in the field of topic modeling. The metric Perplexity [13] is a standard measure of performance for statistical models, which indicates the uncertainty in predicting a 
single word; the model with lower value is better in performance. Perplexity is defined as follows:

$$
\operatorname{Perplexity}\left(M_{\text {test }}\right)=\exp \left\{-\frac{\sum_{m} \ln p\left(w_{m}\right)}{\sum_{m} N_{m}}\right\}
$$

where $M_{\text {test }}$ is a test set with $m$ documents, $w_{m}$ and $N_{m}$ indicate the observed words and number of words in the test document respectively.

Perplexity is used to measure the performance of LDA and MLDA under the same hyperparameters setting, and the result is shown in figure 6. Figure 6 show that the perplexity of MLDA is always lower than LDA, which show that the performance of MLDA is much better than LDA.

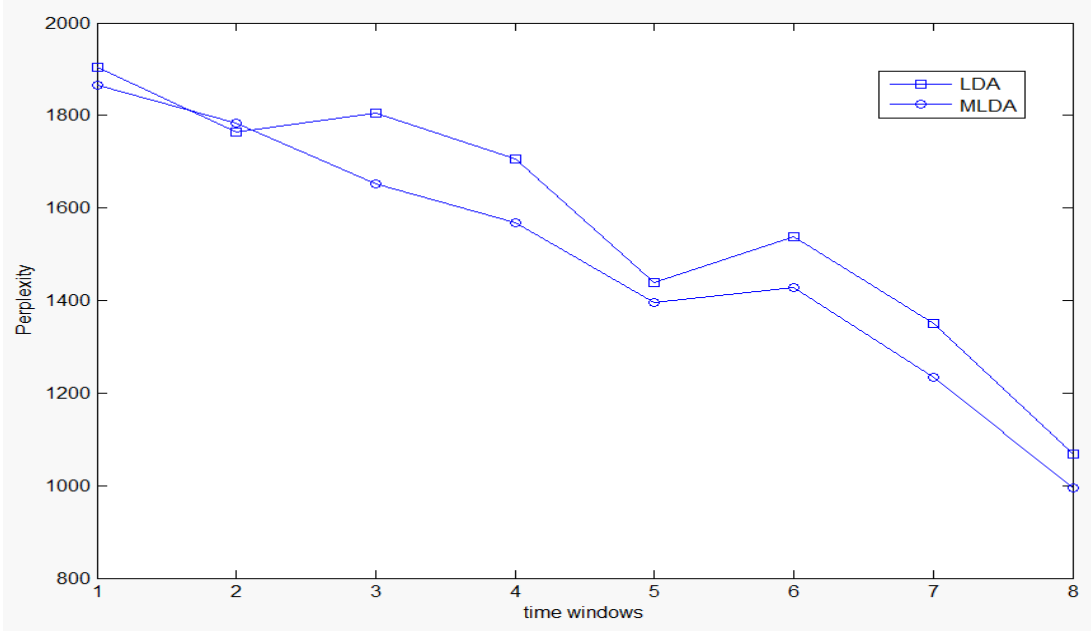

Fig. 7. The performance of MLDA and LDA

\section{Conclusion}

An MLDA model is proposed in this paper, which takes microblog document relation, topic tag and authors' relation into consideration. Based on MLDA, the topic evolution in content and intensity is studied. The experiments on microblog data have shown the effectiveness and efficiency of the approach to depict topic evolution. In future work, we will improve efficiency of this algorithm. Besides, the scalability of the proposed model will be further investigated. 


\section{References}

[1] Allan, J., PaPka, R., Lavrenko, V.: Online new event detection and tracking. In: Proceedings of the 21st Annual International ACM SIGIR Conference on Research and Development in Information Retrieval, pp. 37-45. ACM Press, Melbou

[2] Blei, D., Ng, A., Jordan, M.: Latent Dirichlet allocation. Journal of Machine Learning Research, 993-1022 (2003)

[3] Chu, K., Li, F.: Topic Evolution Based on LDA and Topic Association. Journal of Shanghai Jiao Tong University 44(11), 1496-1500 (2010)

[4] Wang, X., McCallum, A.: Topic over time: A Non-Markov Continuous-Time Model of Topical Trends. In: ACM SIGKDD, pp. 424-433 (2006)

[5] Song, X., Lin, C.Y., Tseng, B.L., et al.: Modeling and predicting personal information dissemination behavior. In: Proceedings of the Eleventh ACM SIGKDD International Conference on Knowledge Discovery in Data Mining, pp. 479-488 (2005)

[6] Mark, S., Tom, G.: Probabilistic Topic Models. Latent Semantic Analysis: A Road to Meaning (2006)

[7] AlSumait, L., Barbará, D., Domeniconi, C.: On-line LDA: adaptive topic models for mining text streams with applications to topic detection and tracking. In: Data Mining, ICDM 2008, pp. 3-12 (2008)

[8] Griffiths, T.L., Steyvers, M.: Finding scientific topics. In: Proceedings of the National Academy of Sciences of the United States of America, pp. 5221-5228 (2004)

[9] Cui, K., Zhou, B., Jia, Y., Liang, Z.: LDA-based Model for Online Topic Evolution Mining. Computer Science 37(11), 156-193 (2010)

[10] Minka, T., Lafferty, J.: Expectation-Propagation for the Generative Aspect Model. Uncertainty in Artificial Intelligence (UAI) (2002)

[11] Blei, D., Lafferty, J.: Visualizing Topics With Multi-Word Expressions. Stat, 1050-1055 (2009)

[12] Hu, Y., Hu, L., Zhang, W.: OLDA-based Method for Online Topic Evolution in Network Public Opinion Analysis. Journal of National University of Defense Technology 34(1), 150-154 (2012)

[13] Blei, D., Lafferty, J.: Dynamic topic models. In: Proceeding of the 23rd in Conference on Machine Learning, pp. 113-120. ACM, New York (2006) 